\title{
Designing Single Sampling Plan using Generalized Poisson Distribution
}

\author{
V. Kaviyarasu ${ }^{1 *}$, V. Devika ${ }^{2}$ \\ ${ }^{1}$ Assistant Professor, Department of Statistics, Bharathiar University, Coimbatore, Tamil Nadu, India \\ ${ }^{2}$ Research Scholar, Department of Statistics, Bharathiar University, Coimbatore, Tamil Nadu, India \\ *Corresponding Author: kaviyarasu@buc.edu.in, Mob: +91-95974 55452
}

Available online at: www.isroset.org

Accepted 15/Aug/2018, Online 30/Aug/2018

\begin{abstract}
This paper introduces a new two-parameter discrete probability distribution through an attribute acceptance sampling plan to develop a sampling plan through the Generalized Poisson Distribution (GPD) called as single sampling plan. The performance measures of the sampling plan are studied through its Operating Characteristic (OC) curve, Operating Characteristic function and its Operating Ratio (OR) values. Designing methods were developed to study the various quality levels namely AQL, LTPD and IQL values and are given for the distribution to protect both the producer and consumer simultaneously. The proposed plan is to support the design of attribute acceptance single sampling plan for lot acceptance with the fraction of non-conforming item modeled by GPD. This distribution is compared with conventional Poisson distribution through its plan parameters. Necessary tables and procedures are constructed with suitable examples to meet the needs of manufacturing industries to study the feasibility of the sampling plan.
\end{abstract}

Keywords - Acceptance Sampling, Single Sampling Plan, Generalized Poisson Distribution,Operating Characteristic Function

\section{INTRODUCTION}

Acceptance sampling plan is concerned with accepting or rejecting a submitted lot quality on the basis of sampling inspection which are widely used in industries to maintain the quality of products. Through the inspection process, larger lots are to be benefited by satisfying the producer and consumer. Moreover, sampling inspection is not a one-time process, which is a continuous process in manufacturing industries. According to Dodge (1969) ${ }^{[1]}$, the major areas of acceptance sampling plans are lot-by-lot sampling inspection methods by attributes, variables, continuous sampling and special purpose plans. This work focuses to develop a new attribute Single Sampling Plan (SSP) using Generalized Poisson Distribution (GPD).

The GPD was discovered by Consol and Jain $(1973)^{[2]}$ which is a two parameter discrete probability distribution, it makes the flexibility of studying in real-time data. A well popularly used single sampling plan by attributes was considered to design and develop a new sampling plan. Our aim is to optimize the sample size and its proportion defective which is to be conceived and tested through the GPD. When manufacturing engineers are interested in improving the quality of products, the traditional distributions like Hyper-geometric, Binomial, Poisson and weighted Poisson distributions were widely studied by several authors towards its industrial applications. The sampling plans are developed to protect both producer and consumer at certain levels. For fulfilling this gap a new distribution in acceptance sampling is studied when the quality engineer expects high quality in a bulky production run up, the generalized case of Poisson distribution is more appropriate distribution than the traditional distribution. The major advantage of this single sampling plan under the condition of GPD is; it has twin properties named as overdispersion as well as under-dispersion according to $\lambda_{2}>0$ or $\lambda_{2}<0$ respectively. An accurate approximation formula for the determination of sampling plan are given in the generalized Poisson case and are studied whenever there exist random fluctuations in product quality.

\section{ASSESSMENT ON LITERATURE}

Most of the literatures in acceptance sampling are developed through SSP using various distributions and designing methods were developed and available in the literature. Cameron (1952) ${ }^{[3]}$ developed SSPs under the conditions of Poisson distribution applying unity value approach whose OC curve passes through two specified points. Hamaker $(1958)^{[4]}$ discussed the problem of finding the optimum sample size by applying various approaches like, usage of process curves, and the minimax principle. Hald $(1967)^{[5]}$ studied the approximate procedures for determining SSPs for specified levels of producer's risk and consumer's risk under the conditions of Poisson, Binomial 
and Hypergeometric distributions. Soundararajan $(1981)^{[6]}$ suggested a procedure to construct tables of SSPs indexed by AQL and AOQL using Poisson distribution which ensure protection to the producer as well as consumer. Govindaraju $(2005)^{[7]}$ presented a procedure to design SSP based on a specified point on the OC curve, which may represent either a good or poor quality lot. Radhakrishnan and Mohana Priya $(2008)^{[8]}$ constructed single and conditional double sampling plan using weighted Poisson distribution as the base line distribution. Liu and Cui (2013) ${ }^{[9]}$ determined single sampling plans under three quality classes of products such as conforming, marginally-conforming and nonconforming through Poisson distribution. Recently, Pradeepa Veerakumari and Azarudheen (2016) ${ }^{[10]}$ evaluate the performance of SSP under the conditions of intervened Poisson distribution. Kaviyarasu and Asif (2018) ${ }^{[11]}$ studied the designing procedure for STDS plan for Zero-inflated Poisson distribution for various quality levels.

\section{THE DEVELOPMENT OF GENERALIZED POISSON DISTRIBUTION}

The design of acceptance sampling plan is studied to balancing the interests of consumer and producer simultaneously. According to Consul and Jain (1973) [[2],[12]] the GPD is well-studied as an alternative to the standard Poisson distribution for a real time data. The GPD is also known as Lagrangian Poisson distribution. In a Generalized Negative Binomial distribution, when $n$ and $\beta$ are large, while $\alpha$ is very small, such that $\alpha=\lambda_{1}, \alpha \beta=\lambda_{2}$, where $\lambda_{1}$ is finite and positive while $\lambda_{2}<1$, the Generalized Negative Binomial distribution can be approximated to the following form

$p_{x}\left(\lambda_{1}, \lambda_{2}\right)=\lambda_{1}\left(\lambda_{1}+x \lambda_{2}\right)^{x-1} \frac{e^{-\left(\lambda_{1}+x \lambda_{2}\right)}}{x !} ; x=0,1,2, \ldots$

In classical approach, designing of a sampling plan passes through the two points on the OC Curve. Here, a Poisson type probability distribution is considered for designing and is defined as GPD. When $\lambda_{2}=0$, the GPD reduces to the Poisson distribution. The parameters are $\lambda_{1}$ and $\lambda_{2}$ which plays important role in both Producer Risk (PR) as well as Consumer Risk (CR) since it posses the twin properties. The mean and variance of the $\operatorname{GPD}\left(x, \lambda_{1}, \lambda_{2}\right)$ are given by

$$
\text { Mean } \mu_{1}^{\prime}=\frac{\lambda_{1}}{1-\lambda_{2}}
$$

and Variance $\mu_{2}=\frac{\lambda_{1}}{\left(1-\lambda_{2}\right)^{3}}$

From equations (2) and (3), it is clear that the mean of the GPD will be smaller than, equal to, or greater than the variance according as the value of $\lambda_{2}$ is positive, zero, or negative.

\section{OPERATING PROCEDURE}

The operating procedure for the new sampling plan will be called as Single Sampling Generalized Poisson Plan (SSGPP). The procedure for this plan is established as follows:

i. Draw a random sample of size $n$ from the lot and determine the number of defectives, $d$ in the sample.

ii. If the defectives $d \leq c$ for a specified interim parameter, then accept the entire lot.

iii. Otherwise reject the lot.

The best discriminating power of any sampling plan can be clearly depicted by its Operating Characteristic Curves (OCC). Here, the operating procedure is used to view OCC by graphically and the probability of lot acceptance against the lot proportion defective for a fixed sample size and their acceptance number are given. Generally, the OCC are characterized by the sample size $(n)$ and acceptance number (c) for the concerned distribution. The PR (Type I error) and the CR (Type II error) can get through directly by analyzing the OCC. Thus it is easy to construct the efficient SSGPP parameters by observing the probability of acceptance against its proportion defectives. In single sampling the decision on acceptance or rejection of a lot is taken on the basis of the quality of one sample from a lot.

\section{THE OPERATING CHARACTERISTIC FUNCTION}

Let us define $N$ as the lot size (possibly infinite), $n$ as the sample size to be drawn in order to make evaluation on lot acceptance and/or rejection, and $c$ be the acceptance number (boundary for the non-conforming units in sample). Let $X$ denotes the number of non-conforming units in the sample. The Probability of acceptance, $P_{a}(p)$, for a given lot proportion defective, $p$, for a SSP is given by

$$
\begin{aligned}
P_{a}(p) & =P[X \leq c] \\
\text { i.e., } \quad P_{a}(p) & =\sum_{x=0}^{c} p_{x}(x ; \lambda)
\end{aligned}
$$

According to Schilling and Neubauer (2009) ${ }^{[[13]]}$, when $n$ is large and $p$ is small, $n / N \leq 0.10$ such that $n p<5$, the proportion defective $p$ is distributed according to the Poisson distribution with mean $n p$.

When the interim parameter plays a role in improving the quality of products exponentially, the mean value of the event $p$ may get changed. To overcome this unusual situation, the Generalized Poisson model is used for 
designing the effective usage of a sampling plan. Thus the probability function for the number of non-conforming units in the sample modeled through the GPD is given by,

$$
P_{a}(p)=\sum_{x=0}^{c} P\left(X=x / \lambda_{1}, \lambda_{2}\right)
$$

That is $P_{a}(p)=\sum_{x=0}^{c} \lambda_{1}\left(\lambda_{1}+x \lambda_{2}\right)^{x-1} \frac{e^{-\left(\lambda_{1}+x \lambda_{2}\right)}}{x !}$

Where $\lambda_{1}=n p$ and $\lambda_{2}$ is the interim parameter. Hence the probability of acceptance can be calculated with the help of above equation for given values of $n p, \lambda_{2}$ and $c$. The Operating Characteristic Curve (OCC) for different sets of parametric values has been carried out using the equation (5).

\section{SIGNIFICANCE OF SINGLE SAMPLING GENERALIZED POISSON PLAN (SSGPP)}

The SSGPP consists of various plan parameters as $n, c$, and $\lambda_{2}$. The probability of acceptance of a lot of LQL $\left(\mathrm{p}_{2}\right)$ quality is restricted to a small value $\beta$ and for protecting the producer an Acceptable Quality Level (AQL) is set up limiting the probability of rejecting for a lot of quality AQL $\left(\mathrm{p}_{1}\right)$ to a small value $\alpha$. The OCC passes through the points $\left(\mathrm{p}_{1}, 1-\alpha\right)$ and $\left(\mathrm{p}_{2}, \beta\right)$. Based on this the plan parameters are designed and one can draw a suitable OCC for the desired discrimination.

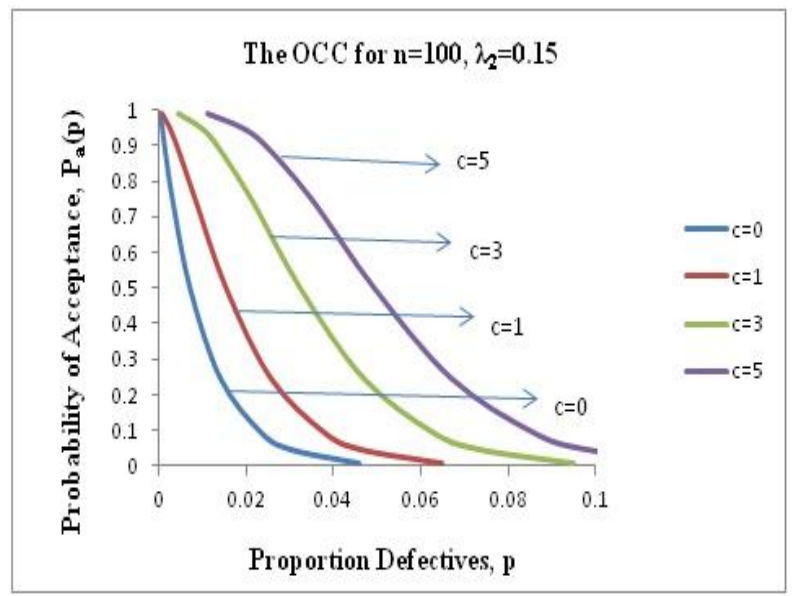

Figure 1. OCC of SSP for different acceptance numbers using GPD

Accordingly, Figure 1 shows the probability of acceptance and its associated risk levels for various acceptance numbers with fixed sample size and interim parameter for GPD. Further, it is clear that for fixed $n$ and $\lambda_{2}$, the probability of acceptance increases for various acceptance numbers and the corresponding probability values for AQL, IQL and LTPD were given in Table 1. The initial approaches employed to design the attribute acceptance sampling plan used the concept of OCC where the risk of plans are studied through the producer and consumer quality levels. All acceptance tests that are destructive must inevitably be performed by sampling inspection since the cost of $100 \%$ inspection is very high.

Table 1. The Proportion Defective values of the SSGPP for different acceptance number

\begin{tabular}{|c|c|c|c|c|c|c|c|}
\hline \multirow{6}{*}{ 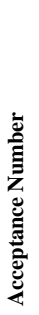 } & & \multicolumn{6}{|c|}{ Probability of Acceptance } \\
\hline & & 0.99 & 0.95 & 0.9 & 0.1 & 0.05 & 0.01 \\
\hline & $\mathrm{c}=0$ & 0.0001 & 0.0005 & 0.0010 & 0.0229 & 0.029 & 0.0458 \\
\hline & $\mathrm{c}=1$ & 0.0006 & 0.0024 & 0.0040 & 0.0374 & 0.0458 & 0.0647 \\
\hline & $c=3$ & 0.0042 & 0.0095 & 0.0131 & 0.0622 & 0.0730 & 0.0950 \\
\hline & $\mathrm{c}=5$ & 0.0109 & 0.0189 & 0.0242 & 0.0852 & 0.0975 & 0.1231 \\
\hline
\end{tabular}

In order to study the effect of sample size with intention to improve the quality, Figure 2 has been obtained by fixing the acceptance number $(c)$ and the interim parameter $\left(\lambda_{2}\right)$.

The OC curves reveal that, the sample size is inversely proportional to the probability of acceptance for higher proportion defectives. On the other hand, producers safe zone is depend on the smaller sample size. Here, the producer as well as consumer will protected simultaneously for reasonably larger sample size through interim parameter. The corresponding probability values for different choice of sample sizes are given in Table 2.

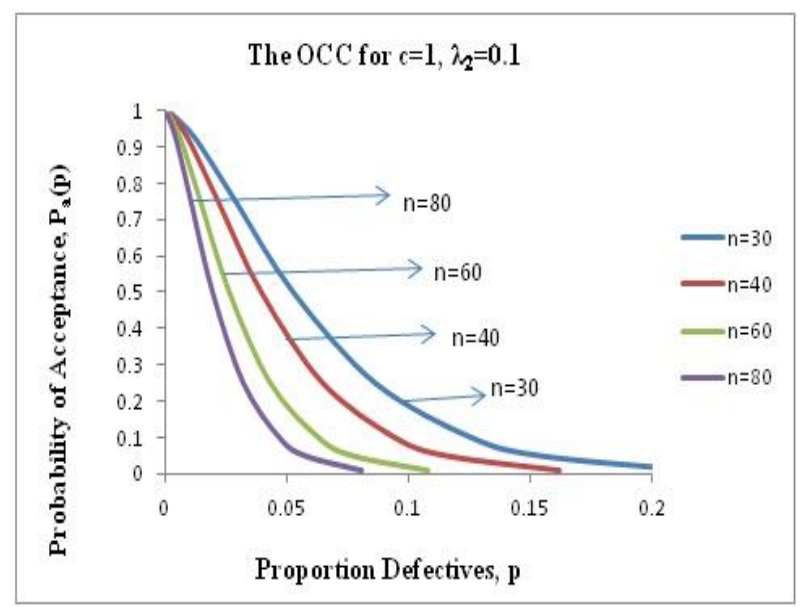

Figure 2. OCC of SSP for different sample numbers using GPD

Table 2. The Proportion Defective values for the SSGPP for different sample sizes

\begin{tabular}{|c|c|c|c|c|c|c|c|}
\hline \multirow{6}{*}{ 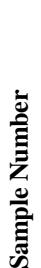 } & & \multicolumn{6}{|c|}{ Probability of Acceptance } \\
\hline & & 0.99 & 0.95 & 0.9 & 0.1 & 0.05 & 0.01 \\
\hline & $\mathrm{n}=\mathbf{3 0}$ & 0.0027 & 0.0090 & 0.0147 & 0.1261 & 0.1547 & 0.2162 \\
\hline & $n=40$ & 0.0020 & 0.0068 & 0.0110 & 0.0946 & 0.1160 & 0.1622 \\
\hline & $n=60$ & 0.0013 & 0.0045 & 0.0074 & 0.0631 & 0.0774 & 0.1081 \\
\hline & $\mathrm{n}=80$ & 0.0010 & 0.0034 & 0.0055 & 0.0473 & 0.0580 & 0.08101 \\
\hline
\end{tabular}


Figure $3 \& 4$ shows a comparison for the PD and GPD through the same acceptance number. The Figure 3 shows that PD and GPD protects producer equally but in consumer point of view more protection is safeguarded through GPD. On comparing, Figure 4 elucidate that GPD saves the consumer more from accepting the bad lot and therefore it makes an alarming sense to the production manager to fulfilling the consumer satisfaction.

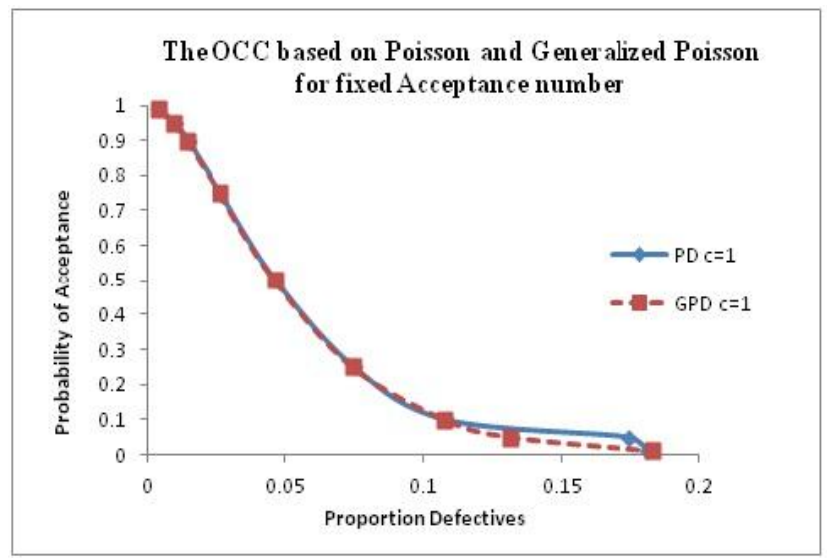

Figure 3. OCC of SSP based on PD and GPD for $c=1$

Table 3. The Proportion Defective values for PD and GPD when $\mathrm{c}=1$

\begin{tabular}{|l|l|l|l|l|l|l|}
\hline & \multicolumn{6}{|c|}{ Probability of Acceptance } \\
\cline { 2 - 7 } & $\mathbf{0 . 9 9}$ & $\mathbf{0 . 9 5}$ & $\mathbf{0 . 9}$ & $\mathbf{0 . 1}$ & $\mathbf{0 . 0 5}$ & $\mathbf{0 . 0 1}$ \\
\hline PD C $=1$ & 0.00410 & 0.00994 & 0.01474 & 0.10803 & 0.17442 & 0.18143 \\
\hline GPD C $=1$ & 0.00384 & 0.00964 & 0.01450 & 0.10773 & 0.13120 & 0.18294 \\
\hline
\end{tabular}

Table 4. The Proportion Defective values for PD and GPD when $c=3$

\begin{tabular}{|l|c|c|c|c|c|c|}
\hline \multirow{2}{*}{} & \multicolumn{6}{|c|}{ Probability of Acceptance } \\
\cline { 2 - 7 } & $\mathbf{0 . 9 9}$ & $\mathbf{0 . 9 5}$ & $\mathbf{0 . 9}$ & $\mathbf{0 . 1}$ & $\mathbf{0 . 0 5}$ & $\mathbf{0 . 0 1}$ \\
\hline PD C $=3$ & 0.00812 & 0.01359 & 0.01739 & 0.06679 & 0.07732 & 0.10014 \\
\hline GPD C $=3$ & 0.00411 & 0.00946 & 0.01317 & 0.06235 & 0.07293 & 0.09579 \\
\hline
\end{tabular}

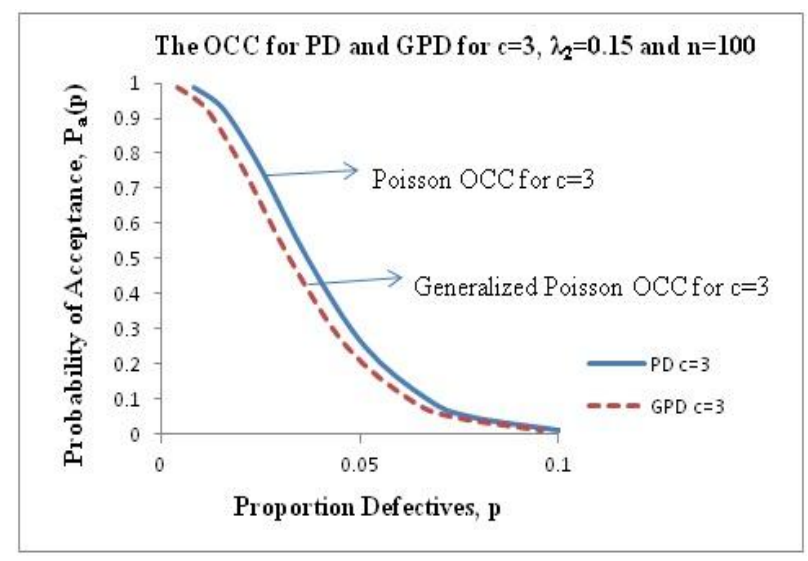

Figure 4. OCC of SSP based on PD and GPD for $c=3$

\section{DESIGNING METHODOLOGY OF SINGLE SAMPLING GENERALIZED POISSON PLAN (SSGPP)}

The SSGPP can be designed through its plan parameters and the development of the plan is obtained by unity value approach elucidated by Schilling and Neubauer $(2009)^{[13]}$. Using this method, the table values are calculated for different set of plan parameters for given plan.

Table 5 provides the $n p_{1}$ and $n p_{2}$ values and Table 6 contains the Operating Ratio (OR) values for various combination of $\alpha$ and $\beta$, such as $[(\alpha=0.05, \beta=0.10)$, $(\alpha=0.05, \beta=0.05),(\alpha=0.05, \beta=0.01),(\alpha=0.01, \beta=0.10)$, $(\alpha=0.01, \beta=0.05)$ and $(\alpha=0.01, \beta=0.01)]$.

The plan parameters for designing the SSGPP can be done by the following procedure:

i) Frame the Acceptable Quality Level (AQL), Lot Tolerance Percent Defectives (LTPD) and the interim parameter $\lambda_{2}$.

ii) Calculate the operating ratio (OR) by using the formula $O R=p_{2} / p_{1}$.

iii) Elect the unity values $n p_{1}$ and $n p_{2}$ from Table-5 which is very close to the calculated OR value.

iv) Determine the sample size as $n=n p_{1} / p_{1}$ and $n=n p_{2} / p_{2}$. Also select the corresponding acceptance number based on the OR value.

v) The SSGPP can formulate the plan parameters as (n, $\left.\lambda_{2}, \mathrm{c}\right)$ for the given interim parameter $\lambda_{2}$.

\section{NUMERICAL ILLUSTRATION}

To design the proposed SSGPP having $p_{1}=0.01, p_{2}=0.06, \alpha=0.05$ and $\beta=0.10$, one has to calculate the operating ratio by the formula $O R=p_{2} / p_{1}=0.06 / 0.01$. The value is 6 , which corresponds to the value of $c=3$ and $\lambda_{2}=0.10$ from Table 6 . From these parameters, the value of $n p_{1}$ and $n p_{2}$ obtained from Table 1 as $n p_{1}=0.8158$ and $n p_{2}=5.3183$ respectively. The SSGPP sample size is calculated as $n=n p_{1} / p_{1}=0.5293 / 0.01=52.93 \quad$ and $/$ or $n=n p_{2} / p_{2}=6.3823 / 0.06=106.37$.

By selecting the large $n$ among the two sample numbers, one can obtain the SSGPP parameters as $(106,0.10,3)$. The relationship between the sample size, interim parameter and their acceptance number are obtained as $\mathrm{n}=106, \lambda_{2}=0.10$ and $\mathrm{c}=3$. That is the maximum number of defects to be permitted is 3 for the acceptance of the lot. 


\section{CONCLUSION}

In this paper, a new attribute acceptance sampling plan is developed under the conditions of GPD to design the plan parameters of single sampling plan and it is designated as Single Sampling Generalized Poisson Plan (SSGPP). The aim is to develop the procedures involved in the sampling plan and investigated through its performance measure values at various quality levels (such as AQL, IQL, LTPD). Further, a comparison between the SSGPP over the conventional Poisson SSP are given. The SSGPP is seemed to be better sampling plan for both producer as well as consumer in terms of its sample size, acceptance number and its associated risks.

The OC function increases disproportionately when the producer and consumer risk are taken into account. The sample size and proportion defectives decreases hence the quality improves. Apparently this new sampling plan helps to improve the manufacturing engineers to maintain the quality consciousness at various levels and this distribution can be extended to other sampling plans available in the literature.

\section{ACKNOWLEDGEMENT}

The authors are happy to acknowledge the comments and helpful suggestions made by an anonymous referee and the editor and our parent University for providing necessary facilities in the Department through DST-FIST and UGCSAP Programmes. We further acknowledge the DST-PURSE (II) for providing funding for the research work.

\section{REFERENCES}

[1] H.F. Dodge, H.G. Romig, "Sampling Inspection Tables", 2nd Edition, New York, Wiley, 1969.

[2] P.C. Consul, G.C. Jain, "A Generalization of the Poisson Distribution", Technometrics, Vol. 15, pp. 791-799, 1973.

[3] J.M. Cameron, "Tables for constructing and for computing the operating characteristics of single sampling plans", Industrial Quality Control, Vol. 9, pp. 37-39, 1952.

[4] H.C. Hamaker, "Some Basic Principles of Sampling Inspection by Attributes", Journal of Applied Statistics, Vol. 17, No.3 pp. 149-159, 1958.
[5] A. Hald, "On the theory of single sampling inspection by attributes based on two quality levels", Review of the International Statistical Institute, Vol. 35, No. 1, pp. 1-29, 1967.

[6] V. Soundararajan, "Single Sampling Attributes Plan Indexed by $A Q L$ and $A O Q L$ ", Journal of Quality Technology, Vol. 13, No. 3, pp. 195-200, 1981.

[7] K. Govindaraju, "Design of Minimum Average Total Inspection Sampling Plans", Communications in Statistics- Simulation and Computations, Vol. 34, No. 2, pp. 485-493, 2005.

[8] R. Radhakrishnan, L. Mohanapriya, "Selection of Single Sampling Plan Using Conditional Weighted Poisson Distribution", Journal of Contemporary Research in Management, pp. 75-79, 2008.

[9] Fangyu Liu, Lirong Cui, "A Design of Attributes Single Sampling Plans for Three-Class Products”, Quality Technology and Quantitative Management, Vol.10, No.4, pp. 369-387, 2011.

[10]K. Pradeepa Veerakumari, S. Azarudheen, "Evaluation of Single Sampling Plan under the Conditions of Intervened Poisson distribution", Communications in Statistics - Simulation and Computation, issue available at http://dx.doi.org/10.1080/03610918.2016.1193196.

[11] V. Kaviyarasu, Asif T. Thottathil, "Designing Special Type Double Sampling Plan for Zero Inflated Poisson Distribution through Various Quality Levels", International Journal of Statistics and Applied Mathematics, Vol.3(4), pp. 44-53, 2018.

[12]P.C. Consul, "Generalized Poisson Distribution: Properties and Applications", Marcel Dekker, New York, 1989.

[13]E.G. Schilling, D.V. Neubauer, "Acceptance Sampling in Quality Control", Boca Raton, CRC Press, 2009.

Table 5. Probability of Acceptance values for SSGPP

\begin{tabular}{|c|c|c|c|c|c|c|c|c|c|c|}
\hline \multirow[b]{2}{*}{$\lambda_{2}$} & \multirow[b]{2}{*}{ c } & \multicolumn{9}{|c|}{ Acceptance Probability } \\
\hline & & 0.99 & 0.95 & 0.90 & 0.75 & 0.50 & 0.25 & 0.10 & 0.05 & 0.01 \\
\hline \multirow{2}{*}{0.01} & 0 & 0.0101 & 0.0510 & 0.1055 & 0.2878 & 0.693 & 1.3832 & 2.3013 & 3.0000 & 4.5402 \\
\hline & 1 & 0.1382 & 0.3470 & 0.5221 & 0.9519 & 1.6682 & 2.6822 & 3.8784 & 4.7231 & 6.5859 \\
\hline
\end{tabular}




\begin{tabular}{|c|c|c|c|c|c|c|c|c|c|c|}
\hline & 2 & 0.4110 & 0.8001 & 1.0849 & 1.7074 & 2.6554 & 3.9005 & 5.2898 & 6.2678 & 8.3192 \\
\hline & 3 & 0.7860 & 1.3343 & 1.7148 & 2.5063 & 3.6422 & 5.0796 & 6.6517 & 7.7219 & 9.9478 \\
\hline & 4 & 1.2145 & 1.9190 & 2.3874 & 3.3330 & 4.6298 & 6.2346 & 7.9451 & 9.1064 & 11.537 \\
\hline & 5 & 1.7258 & 2.5722 & 3.1049 & 4.1754 & 5.6170 & 7.3730 & 9.2240 & 10.464 & 13.025 \\
\hline \multirow{6}{*}{0.05} & 0 & 0.0103 & 0.0510 & 0.1051 & 0.2876 & 0.6929 & 1.3853 & 2.3013 & 2.9926 & 4.5450 \\
\hline & 1 & 0.1051 & 0.3093 & 0.4847 & 0.9127 & 1.6290 & 2.6432 & 3.8288 & 4.6911 & 6.5400 \\
\hline & 2 & 0.3433 & 0.7214 & 1.0054 & 1.6290 & 2.5746 & 3.8211 & 5.2139 & 6.1951 & 8.2334 \\
\hline & 3 & 0.6802 & 1.2120 & 1.5972 & 2.3874 & 3.5231 & 4.9600 & 6.5317 & 7.5963 & 9.8333 \\
\hline & 4 & 1.0807 & 1.7736 & 2.2402 & 3.1680 & 4.4706 & 6.0746 & 7.7915 & 8.9513 & 11.387 \\
\hline & 5 & 1.5332 & 2.3636 & 2.9065 & 3.9708 & 5.4194 & 7.1738 & 9.0408 & 10.264 & 12.827 \\
\hline \multirow{6}{*}{0.10} & 0 & 0.0104 & 0.0520 & 0.1063 & 0.2877 & 0.6935 & 1.3832 & 2.3019 & 2.9926 & 4.5687 \\
\hline & 1 & 0.0800 & 0.2702 & 0.4415 & 0.8675 & 1.5785 & 2.5926 & 3.7842 & 4.6413 & 6.4860 \\
\hline & 2 & 0.2510 & 0.6287 & 0.9118 & 1.5317 & 2.4777 & 3.7205 & 5.1166 & 6.0939 & 8.1591 \\
\hline & 3 & 0.5293 & 1.0760 & 1.4489 & 2.2417 & 3.3763 & 4.8081 & 6.3823 & 7.4385 & 9.7233 \\
\hline & 4 & 0.8708 & 1.5742 & 2.0371 & 2.9749 & 4.2767 & 5.8754 & 7.5888 & 8.7532 & 11.167 \\
\hline & 5 & 1.2994 & 2.1228 & 2.6614 & 3.7237 & 5.1741 & 6.9260 & 8.7627 & 10.005 & 12.509 \\
\hline \multirow{6}{*}{0.15} & 0 & 0.0103 & 0.0513 & 0.1055 & 0.2878 & 0.6927 & 1.3845 & 2.2986 & 2.9896 & 4.5840 \\
\hline & 1 & 0.0637 & 0.2395 & 0.4020 & 0.8249 & 1.5340 & 2.5467 & 2.2986 & 4.5851 & 6.4759 \\
\hline & 2 & 0.1932 & 0.5555 & 0.8249 & 1.4432 & 2.3827 & 3.6249 & 5.0265 & 5.9766 & 8.0338 \\
\hline & 3 & 0.4240 & 0.9507 & 1.3182 & 2.1013 & 3.2285 & 4.6648 & 6.2254 & 7.3000 & 9.5058 \\
\hline & 4 & 0.7000 & 1.4032 & 1.8560 & 2.7845 & 4.0801 & 5.6825 & 7.3837 & 8.5850 & 10.990 \\
\hline & 5 & 1.0921 & 1.8900 & 2.4274 & 3.4847 & 4.9287 & 6.6778 & 8.5238 & 9.7572 & 12.318 \\
\hline
\end{tabular}

Table 6. Operating Ratio values for SSGPP

\begin{tabular}{|c|c|c|c|c|c|c|c|c|c|}
\hline \multirow{2}{*}{$\lambda_{2}$} & \multirow{2}{*}{ c } & \multicolumn{3}{|c|}{$\mathbf{p}_{2} / \mathbf{p}_{1}$ for } & \multirow{2}{*}{$\begin{array}{l}\mathbf{n p}_{1} \text { for } \\
\alpha=0.05\end{array}$} & \multicolumn{3}{|c|}{$\mathbf{p}_{2} / \mathbf{p}_{1}$ for } & \multirow{2}{*}{$\begin{array}{l}\mathrm{np}_{1} \text { for } \\
\alpha=0.01\end{array}$} \\
\hline & & $\begin{array}{r}\alpha=0.05 \\
\beta=0.10\end{array}$ & $\begin{array}{l}\alpha=0.05 \\
\beta=0.05\end{array}$ & $\begin{array}{l}\alpha=0.05 \\
\beta=0.01\end{array}$ & & $\begin{array}{l}\alpha=0.01 \\
\beta=0.10\end{array}$ & $\begin{array}{l}\alpha=0.01 \\
\beta=0.05\end{array}$ & $\begin{array}{l}\alpha=0.01 \\
\beta=0.01\end{array}$ & \\
\hline \multirow{6}{*}{0.01} & 0 & 45.126 & 58.826 & 89.027 & 0.0510 & 228.44 & 297.79 & 450.67 & 0.0101 \\
\hline & 1 & 11.177 & 13.611 & 18.979 & 0.3470 & 28.056 & 34.167 & 47.642 & 0.1382 \\
\hline & 2 & 6.6114 & 7.8338 & 10.398 & 0.8001 & 12.871 & 15.250 & 20.241 & 0.4110 \\
\hline & 3 & 4.9850 & 5.7871 & 7.4552 & 1.3343 & 8.4628 & $9.8 \mathrm{z} 243$ & 12.656 & 0.7860 \\
\hline & 4 & 4.1402 & 4.7454 & 6.0118 & 1.9190 & 6.5420 & 7.4982 & 9.4993 & 1.2145 \\
\hline & 5 & 3.5860 & 4.0680 & 5.0638 & 2.5722 & 5.3448 & 6.0631 & 7.5473 & 1.7258 \\
\hline \multirow{3}{*}{0.05} & 0 & 45.158 & 58.723 & 89.185 & 0.0510 & 223.59 & 290.75 & 441.574 & 0.0103 \\
\hline & 1 & 12.379 & 15.167 & 21.145 & 0.3093 & 36.437 & 44.643 & 62.2385 & 0.1051 \\
\hline & 2 & 7.2278 & 8.5879 & 11.414 & 0.7214 & 15.187 & 18.045 & 23.9824 & 0.3433 \\
\hline
\end{tabular}




\begin{tabular}{|c|c|c|c|c|c|c|c|c|c|}
\hline & 3 & 5.3892 & 6.2676 & 8.1133 & 1.2120 & 9.6030 & 11.168 & 14.4571 & 0.6802 \\
\hline & 4 & 4.3930 & 5.0469 & 6.4201 & 1.7736 & 7.2098 & 8.2830 & 10.5367 & 1.0807 \\
\hline & 5 & 3.8251 & 4.3427 & 5.4270 & 2.3636 & 5.8965 & 6.6945 & 8.36594 & 1.5332 \\
\hline \multirow{6}{*}{0.10} & 0 & 44.276 & 57.561 & 87.876 & 0.0510 & 222.21 & 288.889 & 441.034 & 0.0103 \\
\hline & 1 & 14.007 & 17.180 & 24.008 & 0.3570 & 47.321 & 58.0382 & 81.1057 & 0.1486 \\
\hline & 2 & 8.1389 & 9.6934 & 12.979 & 0.8158 & 20.385 & 24.2783 & 32.5063 & 0.4446 \\
\hline & 3 & 5.9313 & 6.9129 & 9.0363 & 1.3638 & 12.058 & 14.0540 & 18.3709 & 0.8345 \\
\hline & 4 & 4.8206 & 5.5603 & 7.0934 & 1.9679 & 8.7152 & 10.0524 & 12.8241 & 1.2863 \\
\hline & 5 & 4.1279 & 4.7131 & 5.8928 & 2.6069 & 6.7434 & 7.69943 & 9.62671 & 1.7853 \\
\hline \multirow{6}{*}{0.15} & 0 & 44.840 & 58.320 & 89.423 & 0.0513 & 223.32 & 290.456 & 445.361 & 0.0103 \\
\hline & 1 & 9.5991 & 19.148 & 27.044 & 0.2395 & 36.102 & 72.0148 & 101.711 & 0.0637 \\
\hline & 2 & 9.0485 & 10.759 & 14.462 & 0.5555 & 26.018 & 30.936 & 41.5846 & 0.1932 \\
\hline & 3 & 6.5479 & 7.6782 & 9.9983 & 0.9507 & 14.681 & 17.2149 & 22.4168 & 0.4240 \\
\hline & 4 & 5.2620 & 6.1182 & 7.8318 & 1.4032 & 10.548 & 12.2643 & 15.6995 & 0.7000 \\
\hline & 5 & 4.5098 & 5.1624 & 6.5174 & 1.8900 & 7.8049 & 8.93431 & 11.2793 & 1.0921 \\
\hline
\end{tabular}

\section{AUTHORS PROFILE}

Dr. V. KAVIYARASU: He is currently working as Assistant Professor in Department of Statistics, Bharathiar University, Coimbatore, Tamil Nadu. He has published more than 25 research papers in reputed international and national journals and conferences and it's also available online. His main research work focuses on Statistical Quality Control, Reliability Theory and Data Mining. He has 15 years of teaching experience and 10 years of research experience.

V. DEVIKA: Research scholar doing Ph.D., programme in the field of Statistical Quality Control and Data Mining from Department of Statistics, Bharathiar University, Coimbatore, Tamil Nadu, India. 